\title{
ACCELERATION USING TOTAL INTERNAL REFLECTION
}

\author{
R.C. Fernow
}

7 June 1991

\section{CENTER FOR ACCELERATOR PHYSICS}

PHYSICS DEPARTRENT

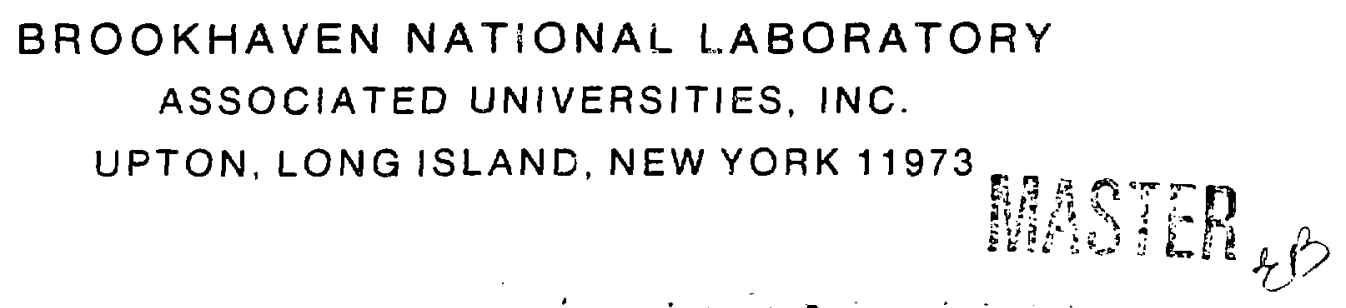




\title{
ALCELERATION USING TOTAL INTERNAL REFLECTION
}

\author{
R.C. Fernow
}

Brookhaven National Laboratory

This report considers the use of a dielectric slab undergoing total internal reflection as an accelerating structure for charged particle beams. We examine the functional dependence of the electromagnetic fields above the surface of the dielectric for polarized incident waves. We present an experimental arrangement for testing the performance of the method, using apparatus under construction for the Grating Acceleration experiment at Brookhaven National Laboratory. 


\section{CONTENTS}

1. Introduction 1

2. Havevector relations 2

3. Polarization of the incident wave 4

4. Solution for incident wave with $\mathrm{H}_{\mathrm{p}}{ }^{2}=0 \quad 5$

5. Solution for incident wave with $E_{\mathrm{p}}{ }^{\mathrm{i}}=0 \quad 8$

6. Forces 11

7. Practical considerations 14

$\begin{array}{ll}\text { Endnotes and references } & 17\end{array}$ 


\section{Introduction}

Methods for using the very large electric fields in laser beams for accelerating charged particles have been under investigation for many years $[1-4]$. In one class of method the particles interact with slow, evanescent waves set up on the surface of an accelerating structure. This method is closely related to that employed in disk loaded linacs powered by RF sources[5]. The axially symmetric cavity fields can be considered as the infinite sum of surface fields. Examples of this technique explicity using external laser beams include acceleration over a metallic grating or acceleration over a dielectric slab undergoing total internal reflection.

The phenomenon of total internal reflection is a well known subject in physical optics[6-9]. Consider a boundary surface with a dielectric of refractive index $n$ on one side and vacuum on the other. Let a plane wave be incident on the boundary from the dielectric side. The wave will be totally reflected back into the dielectric provided the angle $\theta$ with the surface normal exceeds the critical angle $\theta_{\hat{C}}$ given by

$$
\sin \theta_{c}=\frac{1}{n}
$$

However, the solution of the Maxwell equations requires that an evanescent wave exist on the vacuum side of the boundary surface.

The possibility of using the surface fields on a dielectric undergoing total internal reflection to accelerate particles was apparently first suggeated by Lohmann[10] in 1962. He apparently realized that the accelerating field vanishes for relativistic particles in a geometry where the incident wave does not have a component perpendicular to the trajectory of the accelerated particle. We will call this the classical geometry, since it is the one that is customarily employed in discussions of total internal reflection.

The subject has been actively studied by a group at the Yerevan Physics Institute[11-12]. Kheifets[13] gave explicit expressions for the transmitted field components for the case of the classical geometry. He showed that the solutions could be broken into the two cases depending on the polarization of the incident wave. He also studied the conditions for stability for elliptically polarized waves. He found that a net force exists normal to the boundary surface and suggests cancelling it using an external magnetic field. Nagorsky et al[14] showed that the synchronism condition between the incident wave and a particle travelling along the 
surface is equivalent to the condition for Cerenkov radiation. They showed that phase stability required a second magnetic field oriented normal to the particle trajectory and to the dielectric surface.

There have been a number of reviews of new acceleration techniques that also consider the possibility of using total internal reflection $[5,11,12,15]$.

\section{Waverector relations}

Consider the coordinate system shown in Fig. 1. The $x-z$ plane represents the boundary between a region with dielectric constant $n(y<0)$ and free space $(y>0)$. The normal vector to the boundary surface lies along the $g$ direction. A plane wave $k^{1}$ is incident from the dielectric side. The angle $\theta$ is the polar angle between $\mathrm{k}^{2}$ and the $+y$ direction, while the angle $\phi$ is the azimuchal angle between the projection of $k^{1}$ on the $x-z$ plane and the $-x$ axis.

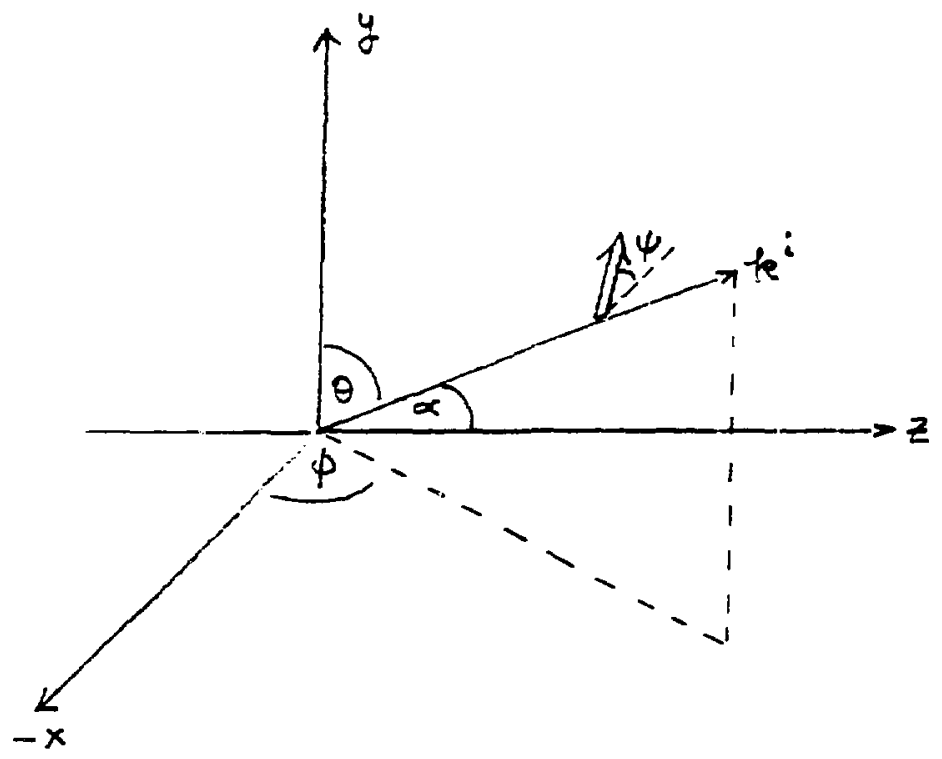

Fig. 1 Coordinate system

Our goal will be to produce suitable electromagnetic field configurations in order to accelerate a particle along the 2 direction. 
TOTAL INTERNAL REFLECTION $/ 3$

The electric field of the incident wave can then be written as

$$
\left.\vec{E}^{1}=\vec{E}_{0}^{1} \exp \left[i k_{1}(-x \sin \theta \cos \phi)+y \cos \theta+z \sin \theta \sin \phi\right)-i \omega t\right]
$$

where

$$
k_{1}=\frac{2 \pi}{\lambda_{1}}=\frac{2 \pi n}{\lambda_{0}}
$$

We will use the SI system of units in this report. The quantity $\lambda_{1}$ is the wavelength of the radiation inside the dielectric while $\lambda_{0}$ is the wavelength in free space.

There must be a reflected wave inside the dielectric that makes an equal angle with respect to the suriace normal. Thus the electric field of the reflected wave can be written

$$
\left.\vec{E}^{r}=\vec{E}_{0}^{r} \exp \left[i k_{1}(-x \sin \theta \cos \phi)-y \cos \theta+2 \sin \theta \sin \phi\right)-i \omega t\right]
$$

We wrice the expression for the electric field of the transmitted wave that exists on the free space side of the boundary in the general form

$$
E^{t}=\vec{E}_{0}^{t} \exp \left[i k_{2 x} x+i k_{2 y} y+i k_{22} z-i \omega t\right]
$$

The boundary plane is uniform in the $x$ and $z$ directions. Thus in order that the combination of waves always staisfy the boundary conditions for all time and for any value of $x$ or $z$ the wavevector components of the transmitted wave along $x$ and $z$ must be identical to those for the incident and reflected waves. Thus we have

$$
\begin{aligned}
& k_{2 x}=-k_{1} \sin \theta \cos \phi \\
& k_{2 z}=k_{1} \sin \theta \sin \phi
\end{aligned}
$$

We can find an expression for $k_{q 7}$ by substituting Eq. 5 for $E^{t}$ into the wave equation

$$
\nabla^{2} \vec{E}^{t}-\epsilon_{0} \mu_{\circ} \frac{\partial^{2}}{\partial t^{2}} \vec{E}^{t}=0
$$

The result is

$$
k_{2 y}= \pm i k_{1} A
$$

where the parineter $A$ is defined as

$$
A=\sqrt{\sin ^{2} \theta-\frac{1}{n^{2}}}
$$

Recall that this derivation is only valid for the conditions of 
total internal reflection, where $\sin \theta>1 / \mathrm{n}$, so $A$ is a real, positive quantity. We have to choose the positive sign in Eq. 8 so that the electric field strength does not grow without bound as we move away from the boundary surface. Thus the transmitted electric field can be written as

$$
\vec{E}^{t}=\vec{E}_{0}^{t} \exp \left[-k_{1} A y\right] \exp \left[i k_{1}(-x \sin \theta \cos \phi)+z \sin \theta \sin \phi\right)-i \omega t f^{10}
$$

The phase velocity of the transmitted electric field along the particle trajectory is

$$
v_{P}=\frac{\omega}{k_{2 z}}=\frac{c}{n \sin \theta \sin \phi}
$$

We see that it is possible to make the particle move in a field of constant phase provided that we choose the index of refraction and the incident angles in such a way that the condition

$$
n \sin \theta \sin \phi=\frac{1}{\beta}
$$

is satisfied, where $\beta$ is the particle velocity in units of $c$. Examining the angles drawn in Fig, 1, we see that the space angle $a$ between $k^{i}$ and the $z$ axis satisfies the relation

$$
\cos \alpha=\sin \theta \sin \phi
$$

Thus the electromagnetic wave and the particle satisfy the same condition required for the production of Cerenkov radiation [14]

$$
\cos \alpha=\frac{1}{n \beta}
$$

\section{Polarization of the incident wave}

Consider a unit vector $V$ that is constrained to be perpendicular to the incident wavevector $\mathrm{k}^{1}$. We represent the orientation of $V$ by using an azimuthal angle as shown in Fig. 1 . Then we can use the Euler angle transformation[16] to write the components of $V$ as

$$
\begin{aligned}
& V_{x}=\sin \phi \cos \psi+\cos \theta \cos \phi \sin \psi \\
& V_{Y}=\sin \theta \sin \downarrow \\
& V_{z}=\cos \phi \cos \psi-\cos \theta \sin \phi \sin \psi
\end{aligned}
$$

$V$ can represent either the $E$ or the $H$ vector of the incident wave. Note that for a given value of the incident angle $\theta$, it is always possible to choose an orientation angle such that $v_{p}$ is 0 . This allows us to look at two simpler classes of problems: one with $E_{\varphi}^{i}$ $=0$ and one with $\mathrm{H}_{\bar{\gamma}}{ }^{2}=0$. 
4 Solution for incident wave with $\mathrm{H}_{\mathrm{T}}{ }^{\mathrm{i}}=0$

In this case we let $V$ in Eq. 15 represent $H^{1}$. Choosing $=0$ gives the condition $H_{p}:=0$. The electric field can then be represented by $V(t=-\pi / 2)$. The incident wave is therefore given by

$$
\begin{aligned}
& H_{X}^{i}=H_{0}^{i} \sin \phi \\
& H_{Y}^{1}=0 \\
& H_{Z}^{i}=H_{0}^{1} \cos \phi \\
& E_{X}^{i}=-E_{0}^{i} \cos \theta \cos \phi \\
& E_{Y}^{1}=-E_{0}^{1} \sin \theta \\
& E_{Z}^{1}=E_{0}^{1} \cos \theta \sin \phi
\end{aligned}
$$

At the boundary surface the fields must satisfy the tangential boundary conditions

$$
\begin{aligned}
H_{o}^{i} \sin \phi+H_{o X}^{r} & =H_{o x}^{t} \\
H_{o}^{i} \cos \phi+H_{o z}^{r} & =H_{o z}^{t} \\
-E_{o}^{i} \cos \theta \cos \phi+E_{o x}^{r} & =E_{o x}^{t} \\
E_{o}^{I} \cos \theta \sin \phi+E_{o z}^{I} & =E_{o z}^{t}
\end{aligned}
$$

The boundary conditions for the normal components are

$$
\begin{aligned}
e_{1}\left(-E_{o}^{I} \sin \theta+E_{o Y}^{I}\right) & =e_{o} E_{o Y}^{t} \\
H_{o Y}^{T} & =H_{o Y}^{t}
\end{aligned}
$$

where we have assumed that the permeabilty of region 1 is also $\mu_{0}$. These 6 equations have a total of 12 unknowns so that 6 of the Maxwells equations are required in addition to solve for the unknowns.

\section{Reflected wave}

The wavevectors are shown schematically in the classical plane in Fig. 2. The solution of the set of equations gives 6 complex expessions for the components of the reflected wave. The magnitudes of the magnetic field components are

$$
\begin{aligned}
& H_{o x}^{Z}=H_{0}^{1} \sin \phi \\
& H_{0 Y}^{Z}=0 \\
& H_{0 Z}^{Z}=H_{0}^{I} \cos \phi
\end{aligned}
$$




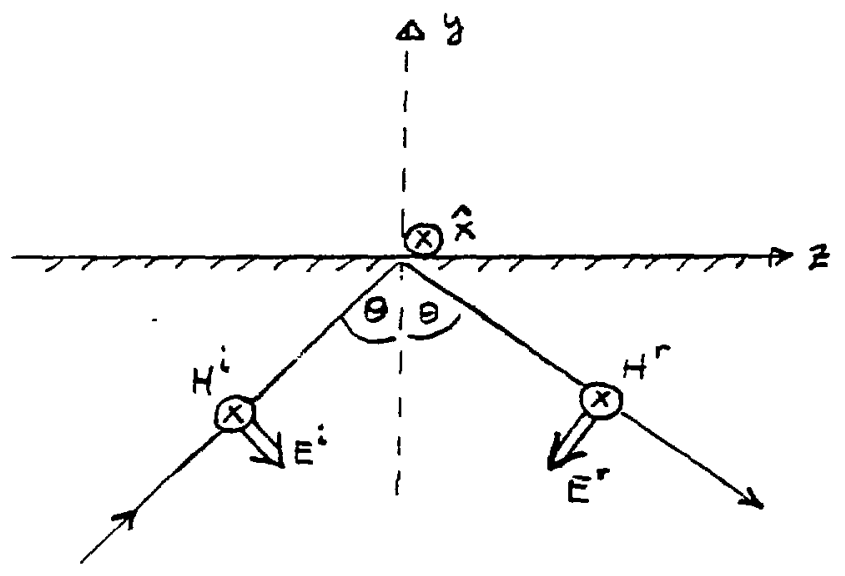

Fig. 2 Field orientation near the boundary for $H_{T}{ }^{i}=0$ polarization.

As we expect the magnitude of $\mathrm{H}^{2}$ is just $\mathrm{H}_{0}{ }^{1}$. Each component has a phase

$$
\tan \Sigma=\frac{-2 n^{2} A \cos \theta}{\cos ^{2} \theta-B^{4} A^{2}}
$$

with respect to the incident wave. The magnitudes of the electric field components are

$$
\begin{aligned}
& E_{0 X}^{I}=E_{0}^{1} \cos \theta \cos \phi \\
& E_{O Y}^{Z}=-E_{0}^{i} \sin \theta \\
& E_{0 Z}^{I}=-E_{0}^{1} \cos \theta \sin \phi
\end{aligned}
$$

The total electric field has the magnitude $E_{0}{ }^{i}$ as expected and each of the components also has the phase $\Sigma$ with respect to the incident wave. 


\section{Transmitted wave}

The magnitudes of the surface electric field components are

$$
\begin{aligned}
& E_{o X}^{c}=\frac{-2 E_{o}^{i} n^{2} A \cos \theta \cos \phi}{\sqrt{\cos ^{2} \theta+n^{6} A^{2}}} \\
& E_{o Y}^{t}=\frac{-2 E_{0}^{i} n^{2} \sin \theta \cos \theta}{\sqrt{\cos ^{2} \theta+n^{4} A^{2}}} \\
& E_{o Z}^{c}=\frac{2 E_{0}^{1} n^{2} A \cos \theta \sin \phi}{\sqrt{\cos ^{2} \theta+n^{4} A^{2}}}
\end{aligned}
$$

The $x$ and $z$ components have the phase relation

$$
\tan \theta=\frac{\cos \theta}{n^{2} A}
$$

with respect to the incident wave. The $y$ component is $90^{\circ}$ out of phase and thus has the phase $(\Omega+\pi / 2)$. The magnitudes of the surface magnetic field components are

$$
\begin{aligned}
& H_{o X}^{t}=\frac{2 H_{0}^{i} \cos \theta \sin \phi}{\sqrt{\cos ^{2} \theta+n^{4} A^{2}}} \\
& H_{o Y}^{t}=0 \\
& H_{o z}^{t}=\frac{2 H_{o}^{1} \cos \theta \cos \phi}{\sqrt{\cos ^{2} \theta+n^{4} A^{2}}}
\end{aligned}
$$

All of the magnetic field components have the phase $(\Omega+\pi / 2)$. 
5 Solution for incident wave with $E_{\eta}^{i}=0$

In this case we let $V$ in Eq. 15 represent $E^{i}$. Choosing $=0$ gives the condition $E_{q}{ }^{1}=0$. The magnetic field can then be represented by $V(1=+\pi / 2)$. The incident wave is therefore given by

$$
\begin{aligned}
& H_{X}^{i}=H_{0}^{1} \cos \theta \cos \phi \\
& H_{Y}^{1}=H_{0}^{1} \sin \theta \\
& H_{Z}^{i}=-H_{0}^{i} \cos \theta \sin \phi \\
& E_{X}^{i}=E_{0}^{i} \sin \phi \\
& E_{Y}^{1}=0 \\
& E_{Z}^{i}=E_{0}^{i} \cos \phi
\end{aligned}
$$

At the boundary surface the fields must satisfy the tangential boundary conditions

$$
\begin{aligned}
H_{o}^{i} \cos \theta \cos \phi+H_{o x}^{r} & =H_{o x}^{t} \\
-H_{o}^{i} \cos \theta \sin \phi+H_{o z}^{r} & =H_{o z}^{t} \\
E_{o}^{i} \sin \phi+E_{o x}^{z} & =E_{o X}^{t} \\
E_{o}^{i} \cos \phi+E_{o z}^{z} & =E_{o z}^{t}
\end{aligned}
$$

The boundary conditions for the normal components are

$$
\begin{aligned}
e_{1} E_{O Y}^{I} & =e_{o} E_{O Y}^{t} \\
H_{o}^{t} \sin \theta+H_{O Y}^{r} & =H_{O Y}^{t}
\end{aligned}
$$

where we have assumed that the permeabilty of region 1 is also $\mu_{0}$. These 6 equations have a total of 12 unknowns so that 6 of the Maxwells equations are required in addition to solve for the unknowns.

Reflected wave

The wavevectors are shown schematically in the classical plane in Fig. 3. The solution of the set of equations gives 6 complex 
expessions for the components of the reflected wave.

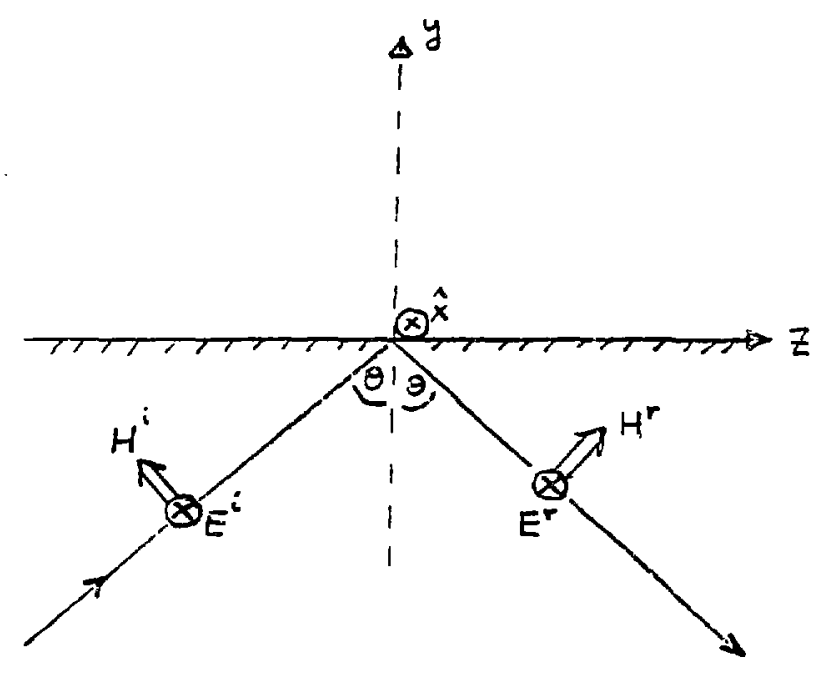

Fig. 3 Field orientation near the boundary for $E_{p}^{i}=0$ polarization.

The magnitudes of the magnetic field components are

$$
\begin{aligned}
& H_{o X}^{z}=-H_{0}^{i} \cos \theta \cos \phi \\
& H_{O Y}^{z}=H_{0}^{i} \sin \theta \\
& H_{o Z}^{T}=H_{0}^{i} \cos \theta \sin \phi
\end{aligned}
$$

As we expect the magnitude of $\mathrm{H}^{l}$ is just $\mathrm{H}_{0}{ }^{\mathrm{i}}$. Each component has a phase

$$
\tan \Pi=\frac{-2 A \cos \theta}{\cos ^{2} \theta-A^{2}}
$$

with respect to the incident wave. The magnitudes of the electric field components are

$$
\begin{aligned}
& E_{0 X}^{I}=E_{0}^{i} \sin \phi \\
& E_{0 Y}^{I}=0 \\
& E_{0 z}^{I}=E_{0}^{i} \cos \phi
\end{aligned}
$$

The total electric field has the magnitude $E_{0}{ }^{i}$ as expected and each of the components also has the phase II with respect to the incident 
wave.

\section{Transmitted wave}

The magnitudes of the surface electric field components are

$$
\begin{aligned}
& E_{0 X}^{t}=\frac{2 E_{0}^{i} n \cos \theta \sin \phi}{\sqrt{n^{2}-1}} \\
& E_{0 Y}^{t}=0 \\
& E_{0 Z}^{t}=\frac{2 E_{0}^{i} n \cos \theta \cos \phi}{\sqrt{n^{2}-1}}
\end{aligned}
$$

The electric field components have the phase relation

$$
\tan T=-\frac{A}{\cos \theta}
$$

with respect to the incident wave. The magnitudes of the surface magnetic field components are

$$
\begin{aligned}
& H_{0 X}^{t}=\frac{2 H_{0}^{1} n A \cos \theta \cos \phi}{\sqrt{n^{2}-1}} \\
& H_{0 y}^{t}=-\frac{H_{0}^{i} n \sin \theta \cos \theta}{\sqrt{n^{2}-1}} \\
& H_{0 Z}^{t}=\frac{-2 H_{0}^{1} n A \cos \theta \sin \phi}{\sqrt{n^{2}-1}}
\end{aligned}
$$

The $x$ and $z$ components of the magnetic field components have the phase $(F+\pi / 2 j$. The $y$ component of the magretic field has the phase $\mathbf{Y}$. 
6 Forces

We consider the forces on a relativistic particle with charge e travelling along the $z$ axis.

$$
\begin{aligned}
& F_{Y}=e\left(E_{Y}-c B_{Y}\right) \\
& F_{Y}=e\left(E_{Y}+c B_{Y}\right) \\
& F_{Z}=\frac{d W}{d z}=e E_{Z}
\end{aligned}
$$

The force component $F_{2}$ gives the rate of change of the particle's energy with distance.

$\underline{E}_{p}=0$ polarization

$$
\begin{aligned}
& F_{x}=\frac{2 e E_{0}^{i} n \cos \theta}{\sqrt{n^{2}-1}}(\sin \phi-\sin \theta) e^{1 \%} G \\
& F_{Y}=\frac{2 i e E_{0}^{i} n A \cos \theta \cos \phi}{\sqrt{n^{2}-1}} e^{i F} G \\
& F_{z}=\frac{2 e E_{o}^{i} n \cos \theta \cos \phi}{\sqrt{\Omega^{2}-1}} e^{i \nabla} \mathrm{G}
\end{aligned}
$$

where

$$
G=\exp \left[-k_{1} A y\right] \exp \left[i k_{1}(-x \sin \theta \cos \phi+z \sin \theta \sin \phi)-i \omega t\right]
$$

We see that the surface accelerating field can never exceed a limiting value of 2 . All field components decrease exponentially with distance above the surface according to Eq. 10. Higher values of $n$ more closely approach the limiting value. Because of the symmetry of $\theta$ and $\phi$ in $F_{l}$, the maximum acceleration rate will be obtained with $\theta=\phi$. Note that the condition $\theta=\phi$ also causes the force in the $x$ direction to vanish. There is a nonvanishing vertical force perpendicular to the surface, but it is $90^{\circ}$ out of phase with the acceleration. Since the vertical force is proportional to $A$, it vanishes at the critical angle.

$\underline{H}_{p}{ }^{i}=0$ polarization

$$
\begin{aligned}
& F_{x}=\frac{-2 e E_{0}^{i} n^{2} A \cos \theta \cos \phi}{\sqrt{\cos ^{2} \theta+n^{4} A^{2}}} e^{i \theta} G \\
& F_{Y}=\frac{2 i e E_{0}^{i} \cos \theta}{\sqrt{\cos ^{2} \theta+n^{4} A^{2}}}\left(\sin \phi-n^{2} \sin \theta\right) e^{i \theta} G \\
& F_{Z}=\frac{2 e E_{0}^{1} n^{2} A \cos \theta \sin \phi}{\sqrt{\cos ^{2} \theta+n^{4} A^{2}}} e^{10} G
\end{aligned}
$$


The accelerating field in this case is considerably more complicated than before. The optimal angles for peak acceleration are calculated numerically in the table below. Note that the force in the $y$ direction vanishes if $\sin (\phi)=n^{2} \sin (\theta)$. However, this is not the same condition required for optimum accceleration.

Table 1 gives examples of the optimal angles and peak surface forces in units of eE for three materials. Note that for a given material it is possible to optimize the values of the incident angles such that the peak acceleration rate is the same for both polarizations. It thus appears that the peak surface accelerating field enhancement will always be less than 2 . We have imposed the constraint

$$
\sin \theta \sin \phi=1
$$

required for synchronism with a relativistic particle. 


\begin{tabular}{|c|c|c|c|}
\hline Table 1 & \multicolumn{3}{|c|}{ Accelerating Forces } \\
\hline material & quartz & ZnSe & $\mathrm{Ge}$ \\
\hline$\underline{\mathbf{n}}$ & 1.54 & 2.40 & 4.00 \\
\hline$\theta_{f}$ & 40.49 & 24.62 & 14.48 \\
\hline \multicolumn{4}{|c|}{$E_{7}^{i}=0$ polarization } \\
\hline$\theta$ & 53.69 & 40.20 & 30.00 \\
\hline$\Phi$ & 53.69 & 40.20 & 30.00 \\
\hline$F_{x}$ & 0 & 0 & 0 \\
\hline$F_{7}$ & 0.440 & 0.633 & 0.671 \\
\hline $\mathrm{F}_{2}$ & 0.922 & 1.284 & 1.549 \\
\hline \multicolumn{4}{|c|}{$\mathrm{H}_{p}{ }^{\mathrm{i}}=0$ polarization } \\
\hline$\theta$ & 48.00 & 29.00 & 16.00 \\
\hline$\phi$ & 60.90 & 59.25 & 65.09 \\
\hline$F_{y}$ & -0.513 & $-0.7 \in 3$ & -0.719 \\
\hline$E_{q}$ & -1.094 & -2.020 & -3.220 \\
\hline$E_{2}$ & 0.922 & 1.282 & 1.549 \\
\hline
\end{tabular}




\section{Practical considerations}

Previous authors $[13,14]$ have considered how an actual total internal reflection accelerator could in principle be assembled from a series of crystals, together with external magnetic fields for stability. We want to examine here whether it would be possible to easily (and cheaply) measure the important parameters of the method experimentally using the apparatus from the Grating Acceleration experiment[17] currently under construction at the Accelerator Test Facility (ATF) at Brookhaven National Laboratory[18]. Measured quantities should include the strength of the acceleration gradient, transverse forces, sensitivity to the direction and polarization of the incident wave, and the breakdown strength of the dielectric medium.

Some aspects of a possible experimental arrangement are shown in Fig. 4. We use incident light from the ATF CO, laser. A total energy of up to $100 \mathrm{~mJ}$ should be available in a 6 ps long pulse. A parallel beam is focussed with a cylindrical lens through an observation window in the interaction chamber. The dielectric medium must have at least one flat side and must be transparent to $\mathrm{CO}_{2}$ radiation. Thus we choose to use a $\mathrm{ZnSe}$ prism. The $\mathrm{CO}_{2}$ beam enters the prism face along a normal and strikes the flat bottom surface. The ZnSe prism is held off the mounting plate using four precision spacers. The electron beam axis is within $10 \mu \mathrm{m}$ of and parallel to the bottom surface. The image of the cylindrical lens should be along the electron beam direction.

Unfortunately for this application, the geometrical configuration of the chamber does not allow the use of the optimal incident angles for ZnSe given in Table 1 . The most practical location for an observation port for this purpose has $\theta=65.4^{\circ}$ and $\phi=27.3^{\circ}$. The predicted forces (in units of $e E_{0}{ }^{l}$ ) are given in Table 2 . The transverse forces dominate the acceleration for $\mathrm{H}_{\mathrm{P}}{ }^{\mathrm{l}}=0$ polarization. The other polarization state has a larger acceleration. However, since we had to give up the $\theta=\phi$ condition, it also has a substantial $x$ component of transverse force.

The analysis spectrometer following the interaction chamber will use point to point focusing in the bend $(x-3)$ plane. Thus to lowest order in the beam optics the $F_{y}$ forces simply represent an additional divergence at the source and they will not be measured by the spectrometer. The $F_{\eta}$ force changes the particle's momemtum so the dipole in the spectrometer will spread the trajectories accordingly in the $x$ direction. The spectrometer will use point to parallel focusing in the non-bend $(y-z)$ plane. This causes the distribution of trajectories to be spread out along $y$ at the detector in proportion to the force $F_{\eta}$. 

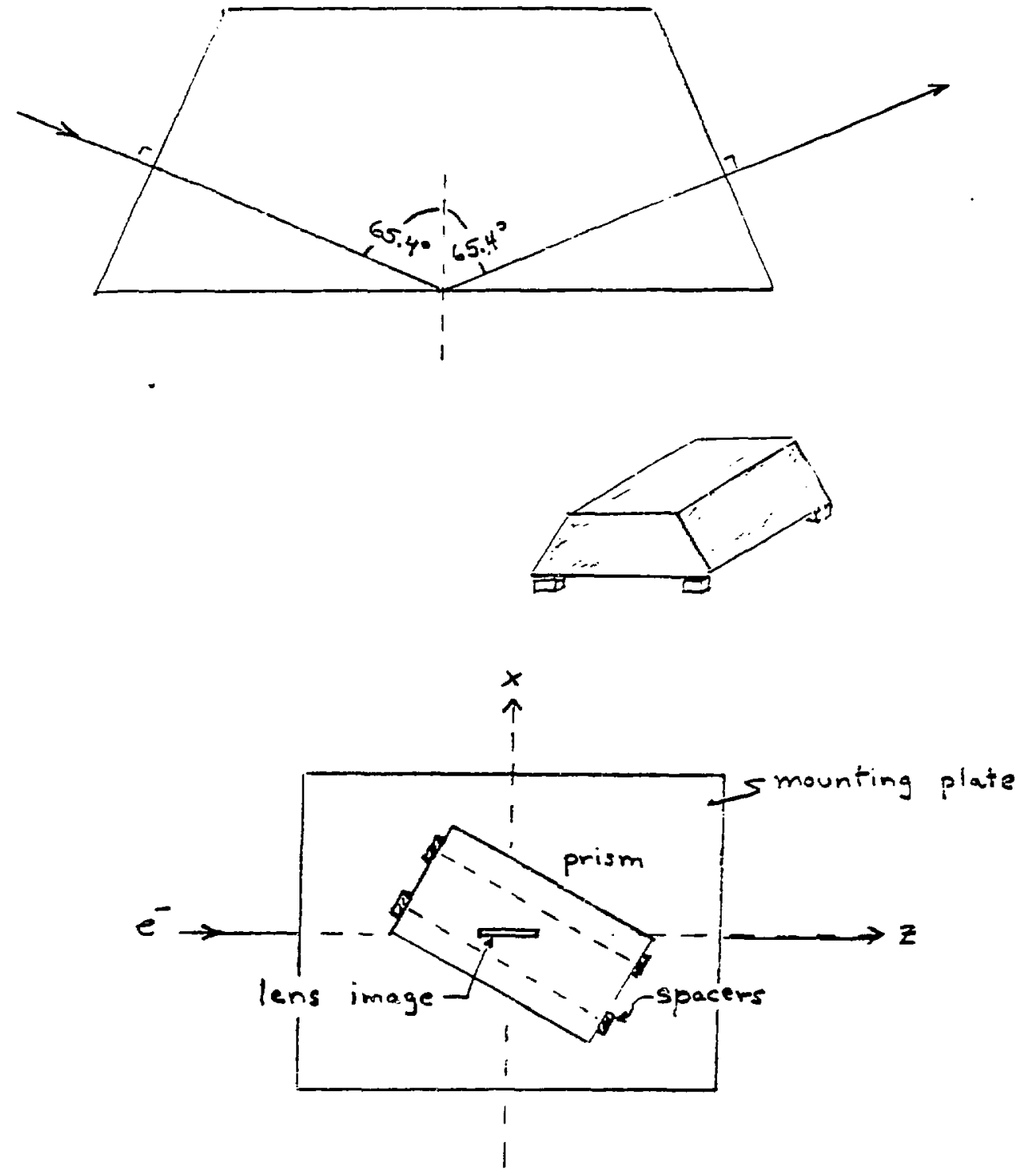

Fig. 4 Experimental arrangement for testing acceleration of electrons passing near a ZnSe prisa. 
TOTAL INTERNAL REFLECTION / 16

\begin{tabular}{|c|c|c|c|}
\hline Table 2 & \multicolumn{3}{|c|}{ Proof of principle performance } \\
\hline material & \multicolumn{3}{|c|}{$\mathrm{ZnSe}$} \\
\hline $\mathrm{n}$ & \multicolumn{3}{|l|}{2.40} \\
\hline$\theta$ & \multicolumn{3}{|l|}{$65.38^{\circ}$} \\
\hline$\phi$ & \multicolumn{3}{|l|}{$27.28^{\circ}$} \\
\hline & $F_{X}$ & $\mathrm{~F}_{\mathrm{p}}$ & $F_{2}$ \\
\hline $\mathrm{H}_{\mathrm{p}}{ }^{1}=0$ & -0.738 & -0.852 & 0.380 \\
\hline$E_{p}{ }^{l}=0$ & -0.413 & 0.658 & 0.815 \\
\hline
\end{tabular}

The performance of the device should be determined chiefly by (1) the strength of electric field that can be tolerated by the ZnSe prism before breakdown and (2) the alignment accuracy for positioning the electron beam parallel to the surface of the prism.

\section{Acknow ledgements}

I would like to thank Stan Ulc, Triveni Srinivasan-Rao, Juan Gallardo and Thomas Tsang for useful discussions. 
[1] P. Channel (ed), Laser Acceleration of Particles, AIP Conf. Proc. 91, 1982 .

[2] C. Joshi \& T. Katsouleas (eds), Laser Acceleration of Particles, AIP Conf. Proc. 130, 1985.

[3] F. Mills (ed), Advanced Accelerator Concepts, AIP Conf. Proc. $156,1987$.

[4] C. Joshi (ed), Advanced Accelerator Concepts, AIP Conf. Proc. 193,1989 .

[5] J. Lawson \& M. Tigner, The physics of particle accelerators, Ann. Rev. Nucl. Part. Sci. 34:99-123, 1984 .

[6] J. Stratton, Electromagnetic Theory, McGraw Hill, 1941, p. 497500 .

[7] P. Lorraine \& D. Corsor, Electromagnetic Fields and Waves, 2nd ed, Freeman, 1979, p. 520-32.

[8] M. Born \& E. Wolf, Principles of Optics, 6th ed., Pergamon Press, 1980, p. $47-51$.

[9] J. Strong, Concepts of Classical Optics, Freeman, 1973, p. 115$119 ; 516-8$.

[10] This is mentioned in J.D. Lawson, Laser accelerators: where do we stand?, in P. Bryant \& J. Mulvey (eds), The generation of high fielcs for particle acceleration to vers high energies, CERN report $85-07,1985$, p. 3-12.

[1 11 ] A.Ts. Amatuni, S.S. Elbakyan, E.M. Laziev, H.A. Nagorsky, M.L. Petrossyan, \& E.V. Sekhpossyan, Development of new methods for charged particle acceleration at Yerevan Physics Institute (review), Yerevan Institute report, 1989.

[12] A.Ts. Amatuni, S.S. Elbakyan, E.M. Laziev, G.A. Nagorsky, M.L. Petrossyan, \& E.V. Sekhpossyan, Development of new methods of charged particle acceleration at Yerevan Physics Institute, Part. Acc. $32: 221-7,1990$.

[13] S.A. Kheifets, Particle motion near a surface on which tota] internal reflection of electromagnetic waves takes place, in M.H. Blewett (ed), Proc. 8th Int. Conf. on High Energy Accelerators, CERN 1971, p. 597-9. 
[14] H.A. Nagorsky, A.Ts. Amatuni, \& W.M. Harutiunian, Resonance acceleration of charged particles by a surface wave arising at total internal reflection, Proc. 12th Int. Conf. on High Energy Accelerators, Fermilab 1983, p. 488-90.

[15] J. Arnesson \& F. Kneubuhl, Future laser driven particle accelerators, Infrared Phys. 25:121-31, 1985 .

[16] H. Goldstein, Classical Mechanics, Addison-Wesley, 1965, p. $107-9$.

[17] W. Chen et al, Proposal for a study of laser acceleratior of electrons using micrograting structures at the $A T F$, BNL report 43465,1989 .

[18] K. Batchelor et al, Operational status of the Brookhaven National Laboratory Accelerator Test Facility, Proc. 1989 Particle Accelerator Conference, p. 273. 